\title{
EFECTOS DE UN PROTOCOLO APLICADO POR ENFERMERAS EN EL CONTROL METABÓLICO A PERSONAS CON DIABETES TIPO 2
}

\section{EFFECTS OF A PROTOCOL FOR NURSES IN APPLIED METABOLIC CONTROL IN PEOPLE WITH TYPE 2 DIABETES}

\author{
Gloria Solano Solano* \\ Ana Emilia Pace ${ }^{* *}$ \\ Cleotilde García Reza ${ }^{* * *}$ \\ Arturo Del Castillo Arreda ${ }^{* * * *}$
}

\begin{abstract}
RESUMEN
El propósito de este estudio cuasi experimental fue evaluar los efectos del control metabólico antes y después de la intervención educativa para pacientes con diabetes tipo 2, en el Estado de Hidalgo, México. Participaron 82 pacientes, se formaron dos grupos: grupo intervención, recibió consultas de enfermería conjuntamente con el equipo multidisciplinario, y grupo control fue con una intervención tradicional. En ambos grupo se determinó peso, talla, niveles séricos, glucosa sanguínea en ayuno, colesterol HDL, LDL, hemoglobina glucosilada, durante seis meses. El análisis de datos se efectuó con la prueba de significación $t$ student $\mathrm{p}=0.05$. Los dos grupos participantes con predominio mujeres, mayores de 50 años, casadas, empleadas y jubiladas, y con un promedio de escolaridad de $5.9 \pm 2.8$ años. Después de la intervención, los promedios de HbA1c disminuyeron en forma significativa en el grupo de intervención; las concentraciones de HbAlc fueron mayores en el grupo control (5.65 \pm 0.99 versus $7.88 \pm 1.95)$. Los resultados de la intervención por enfermería en México facilitan el desarrollo de nuevos programas para el seguimiento permanente ya que mostró un mejor control de niveles de glucemia que los del grupo control.
\end{abstract}

Palabras clave: Diabetes Mellitus tipo 2, educación en salud, enfermería.

\begin{abstract}
The purpose of this quasi-experimental study was to evaluate the effects of metabolic control before and after the educational intervention in patients with type 2 diabetes in the state of Hidalgo, México. 82 patients were enrolled, two groups were created: the intervention group received nursing visits along with the multidisciplinary team, and control group with traditional intervention. In both group, weight, height, serum fasting blood glucose, cholesterol, HDL, LDL, glycasted hemoglobin were determined and measured for six months. Data analysis was performed using the Student $t$ test of significance $p=0.05$. Both groups predominantly female participants, aged 50 years, married, employed and retired, and with an average schooling of $5.9 \pm 2.8$ years. After the intervention, the mean $\mathrm{HbAlc}$ decreased significantly in the intervention group, $\mathrm{HbAlc}$ levels were

\footnotetext{
* Doctora en Enfermería, profesora investigadora de tiempo completo de la Universidad Autónoma del Estado de Hidalgo, E-mail: glorias_4020@yahoo.mx

** Doctora profesora asociada, Escola de Enfermagem de Ribeirão Preto, Universidad de São Paulo Brasil, Centro Colaborador de la OMS para el desarrollo de la Investigación en Enfermería, Brasil. E-mail: aepace@eerp.usp.br

*** Doctora en Enfermería, líder del Cuerpo Académico: Enfermería y cuidado de la Salud, profesor investigador de tiempo completo de la Universidad Autónoma del Estado de México. E-mail: cgarc0506@yahoo.com.mx

${ }^{* * * *}$ Maestro profesor investigador de tiempo completo de la Universidad Autónoma del Estado de Hidalgo, colaborador. E-mail: catles78@hotmail.com
} 
higher in the control group $(5.65 \pm 0.99$ versus $7.88 \pm 1.95)$. The results of the nursing intervention in México facilitate the development of new programs for permanent follow up since it showed better control of blood glucose levels than in the control group.

Key words: Diabetes Mellitus, type 2, health education, nursing.

Fecha recepción:12/09/12 Fecha aceptación: 10/01/13

\section{INTRODUCCIÓN}

La diabetes constituye un grupo heterogéneo de alteraciones metabólicas que presentan hiperglicemia, resultante de fallos en la acción y/o secreción de insulina (1). La hiperglicemia crónica está asociada a daños a largo plazo, disfunciones y alteraciones en los órganos blanco: ojos, riñones, nervios, corazón y vasos sanguíneos (2).

En los últimos 25 años, la prevalencia de diabetes en el mundo aumenta en forma alarmante, no sólo en adultos sino también en niños y adolescentes; alcanza proporciones epidémicas que afectan aproximadamente a 366 millones de personas, lo que representa alrededor de $6 \%$ de la población adulta en el mundo; la proyección de personas con diabetes es impresionante; se estima que para 2030 alcanzará 556 millones $(3,4)$.

La elevada prevalencia de la diabetes tipo 2 (DT2) se atribuye a un complejo conjunto de factores genéticos, sociales y ambientales en los países del mundo; entre estos factores destacan: urbanización, industrialización, crecimiento poblacional, aumento de las expectativas de vida, y envejecimiento de la población; aunado a esto, las alteraciones del estilo de vida, alimentación y sedentarismo favorece el aumento de la morbilidad por DT2 $(5,6)$.

Con la finalidad de brindar a los profesionales de la salud un método sistematizado de detección y tratamiento, además de los recursos académicos para tomar decisiones informadas en la atención, el Centro Internacional de Diabetes (IDC), en Minneapo- lis, Minnesota (USA), desarrolló el protocolo internacional Staged Diabetes Management (Manejo de diabetes por Etapa) (MDE), una guía clínica con "Algoritmos de Decisión" (7) diseñados para clasificar y diagnosticar, definir opciones de tratamiento y monitorear parámetros metabólicos y lipídicos, a través de un algoritmo que establece el tiempo máximo para cada tipo de intervención en tres fases terapéuticas: inicio, ajuste y sostén (8).

El protocolo MDE es evaluado con estudios clínicos y de implementación en más de 200 centros de todo el mundo, con probada eficacia en la reducción de hemoglobina glucosilada (A1c), y disminución de complicaciones crónicas derivadas de la enfermedad; se basa en recomendaciones emitidas por la Asociación Americana de Diabetes (1), la Organización Mundial de la Salud (OMS), la Federación Internacional de Diabetes (2) y otras relacionadas con el manejo de diabetes. Es una guía para organizar la práctica clínica por etapas; éstas inician con ejercicio, nutrición, antidiabéticos orales e insulinas, el objetivo de la guía es mantener cifras de glucosa y parámetros de control metabólico en niveles normales $(7,9)$.

Según las estimaciones en el mundo, los gastos sanitarios por diabetes se han elevado a 465.000 millones de USD en 2011, lo que equivale al $11 \%$ del gasto sanitario total. $\mathrm{Si}$ no se invierte en hacer que los tratamientos sean eficaces para prevenir las complicaciones diabéticas y estén al alcance de todos, se predice que esta cifra aumentará hasta los 595.000 millones de USD para 2030. El Foro Económico Mundial ha identificado 
consistentemente la diabetes (1) como un riesgo mundial para los negocios y las comunidades. Las pérdidas en ingresos nacionales debidas a muertes (en gran parte evitables) por diabetes, enfermedad cardiaca y derrame cerebral son enormes; entre 2005 y 2015 , dichas pérdidas se calcula que alcanzarán los 558.000 millones de USD en China y los 303.000 millones de USD en Rusia (2).

En México el porcentaje de la población que padece diabetes aumenta con la edad. Después de los 50 años, la prevalencia supera el $20 \%$. Esta enfermedad es causa de la mayor demanda de atención médica; principal origen de hospitalización por cardiopatías, enfermedades cerebro-vasculares, insuficiencia renal, y ceguera (8). Además, consume el mayor porcentaje de gasto en las instituciones públicas (alrededor de 20\%), su prevalencia va en aumento, ya que cada año mueren 67 mil personas, así como el surgimiento de 500 mil nuevos casos por causas atribuidas a la diabetes, según la Encuesta Nacional de Salud y Nutrición (5) .

En México, en 2006, expresó una prevalencia de $14.4 \%$. Se estima que 7.3 millones de mexicanos tienen diabetes, de los cuales 3.7 millones conocen su diagnóstico y cerca de $60 \%$ de los casos habían sido diagnosticados hacía cinco o más años y $40 \%$ en el momento de la encuesta. La IDF, sigla en inglés de Federación Internacional de Diabetes, predice que para 2030 el número de mexicanos con diabetes será de 11.9 millones (4).

Es evidente que la mortalidad mexicana de 2005 a 2009, por diabetes, aumentó de 64.54 a 72.18 por cada 100 mil personas (4). El estado de Pachuca Hidalgo supera casi 30 puntos la media nacional, con tasas de 93.81 en 2005 y de 100.78 en 2009. Es así que la diabetes en nuestro país genera un gasto al año de 317 millones de pesos, únicamente para el cuidado de los pacientes; es uno de los porcentajes más elevados del presupuesto en salud $(5,6)$.

Considerando el papel tan importante de la enfermera en la aplicación de un programa de intervención para enfrentar los retos de la alta prevalencia de la diabetes en México, nace la decisión para introducir acciones complementarias sobre el cuidado del paciente con diabetes tipo 2. Con un abordaje para impulsar a las enfermeras a deliberar sobre los programas de trabajo que las coloquen en el centro de las discusiones críticas del cuidado de la salud de estas personas para lograr que un mayor número de pacientes obtengan un control metabólico a largo plazo. Esto representa un nuevo paradigma para la actuación de la enfermera de este tercer milenio.

\section{Objetivo}

Evaluar los efectos del control metabólico antes y después de la intervención educativa para pacientes con diabetes tipo 2 en el Estado de Hidalgo, México.

\section{MATERIAL Y MÉTODO}

Se realizó un estudio cuasi experimental en 82 pacientes con diabetes tipo 2 , con asignación aleatoria a dos grupos de pacientes diabéticos. Para ello se aplicó el protocolo de MDE, habiendo formado dos grupos: Grupo Intervención y Grupo Control, todos ellos atendidos en el centro de salud "Jesús del Rosal" de los Servicios de Salud en Pachuca, Estado de Hidalgo.

El tamaño de la muestra se calculó por medio del Programa de Análisis Epidemiológico de Datos Tabulados EPIDAT V. 3.1; la muestra fue de 38 pares y se adicionó un índice de no respuestas de $14 \%$, obteniendo 39,52 pares, con un total de 82 personas para formar los dos grupos. El grupo de intervención quedó integrado por 41 personas y el control por 41 personas con diabetes tipo 2 , quienes cubrieron un $95 \%$ de las sesiones educativas. 
Criterios de inclusión: personas con DM tipo 2 , sin tratamiento medicamentoso, registradas en el centro de salud, mayores de 18 años, sin patologías que afecten el nivel de comprensión y comunicación.

Criterios de exclusión: embarazadas con diabetes mellitus Tipo 2, registro incompleto y con dificultades para trasladarse, ya que impide participar en el programa educativo.

Antes de iniciar la intervención educativa, se consideraron los resultados de laboratorio registrados en el mes de junio, tanto para el grupo de intervención 41 pacientes y el control con 41. Después de la intervención se emplearon los resultados finales de laboratorio del mes de diciembre de 2008. El grupo control acudió a un programa de educación tradicional, se determinaron estrategias informativas audiovisuales, de $30 \mathrm{~min}$, una vez por semana. El grupo intervención recibió consultas de enfermería en forma conjunta con el equipo multidisciplinario, de acuerdo con las directrices del protocolo MDE de intervención educativa.

La recolección se efectuó en el centro de salud, de junio a diciembre de 2008. Para eso se diseñó un instrumento para evaluar las variables lipídicas (colesterol total, HDL, LDL, triglicéridos, hemoglobina glucosilada) y no lipídicas (peso, talla para construir el Indice de Masa Corporal $($ IMC) $>27$ y <35) y las variables sociodemográficas (edad, sexo, estado civil, escolaridad, ingreso familiar y ocupación).

Para implementar el programa educativo se habilitó al equipo multidisciplinario de salud en el manejo del protocolo $\operatorname{MDE}(7,8)$, logrando un trabajo consensuado, en forma permanente, para las intervenciones farmacológicas, nutricionales, actividad física, automonitoreo y educación para la salud, de acuerdo con los algoritmos del tratamiento derivado de dicho protocolo.

El desarrollo del programa educativo fue una vez por semana, en un aula del centro de salud, con una duración máxima de $2 \mathrm{~h}$ por sesión, en un horario preestablecido, de 08.00 a 10.00 h A.M., para concluir con 16 sesiones educativas, coordinado por enfermería, médico, nutriólogo y psicólogo. Los contenidos del curso se impartieron durante los primeros $30 \mathrm{~min}$, con las técnicas de enseñanza-aprendizaje, se basaron en las directrices del protocolo MDE (7). Se utilizaron metodologías educativas, de participación, que incluyeron procesos de animación para crear una atmósfera natural, sencilla, con discusiones de grupo relacionadas con su experiencia cotidiana y se realizaron actividades demostrativas, con: carteles, figuras, diapositivas, folletos y equipo de demostración, como: jeringas, agujas, y monitor de glucosa. Posterior a la sesión educativa se realizaban ejercicios aeróbicos de una $\mathrm{h}$, y los $30 \mathrm{~min}$ restantes fueron para aclarar dudas y registrar la información de los participantes.

El programa de trabajo se efectuó en tres fases terapéuticas: inicio, ajuste y sostén.

1. La fase de inicio la constituyó la aplicación del cuestionario y la toma de variables lipídicas.

2. La fase de ajuste fue la teorización, basada en los aspectos operativos de la diabetes, tratamiento, hábitos de alimentación y ejercicio.

3. La fase de sostén fue todo lo relacionado con el cotejo de los hábitos de comportamiento dietético, ejercicio, tratamiento y el control metabólico.

Para el manejo del grupo control, sólo recibieron la consulta médica tradicional y de rutina en el consultorio médico del centro de salud.

En ambos grupos se efectuaron mediciones mensuales de las medidas antropométricas IMC $>27$ y $<35$ y los niveles séricos glucosa sanguínea en ayuno (1-3); se consideraron alterados los siguientes valores: glicemia $>100 \mathrm{mg} / \mathrm{dL}$, colesterol total $\geq 200 \mathrm{mg} / \mathrm{dL}$, colesterol HDL $<45 \mathrm{mg} /$ $\mathrm{dL}$, colesterol LDL $\geq 130 \mathrm{mg} / \mathrm{dL}$, triglicé- 
ridos $>150 \mathrm{mg} / \mathrm{dL}$ y hemoglobina glucosilada $(\mathrm{A} 1 \mathrm{c}<7 \%)$. Estos se efectuaron en una unidad hospitalaria durante los seis meses de la intervención educativa. Las mediciones se realizaron al inicio del programa para continuar en forma mensual y al finalizar se procedió a realizar la medición de las variables propuestas a ambos grupos, también se les aplicó una última medición del instrumento para constatar la diferencia entre ambos resultados; aclaramos que vigilamos sigilosamente a los participantes y no tuvimos ninguna deserción.

En la organización y análisis de la información se diseñó una base de datos con el programa SPSS, versión 18. Los datos capturados antes y después de la aplicación del protocolo fueron presentados en cifras absolutas y porcentajes. Para determinar la presencia de diferencias estadísticas entre los dos momentos evaluativos se empleó la prueba t de student de una cola. Se aceptó como criterio un nivel de significación $<0.05$.
Este estudio fue aprobado por el Comité de Ética e Investigación, el subdirector del Departamento de Investigación de Salud en Pachuca, Hidalgo México y la Escuela de Enfermería y Obstetricia de Celaya, Universidad de Guanajuato. Se basó, de forma ética, en la Ley General de Salud de México (10), en lo que respecta a la investigación científica en seres humanos (artículo 21, incisos I, II, III, IV y V).

\section{RESULTADOS}

Los datos descriptivos de las variables demográficas del estudio fueron los siguientes: el grupo de intervención se conformó por 41 pacientes. El (70.7\%) del sexo femenino, con edad promedio $60.4 \pm 10.5$ años, el estado civil: $(82.5 \%)$ casadas, con una escolaridad promedio de $5.9 \pm 2.8$ años, la ocupación se distribuyó entre empleadas (56\%) y amas de casa (31\%), el promedio de duración de la enfermedad de $4.1 \pm 2.8$ años (Tabla 1 ).

Tabla 1. Característica sociodemográfica antes y después, según grupos intervención y control. Pachuca Hidalgo, México, 2007- 2008.

\begin{tabular}{lccc}
\hline \multicolumn{1}{c}{ Características } & Grupo intervención & Grupo control \\
\hline Tamaño de la muestra & & 41 & 41 \\
Sexo & & & \\
& Femenino & $70.7 \%$ & $51.2 \%$ \\
& Masculino & $29.3 \%$ & $48.3 \%$ \\
Promedio de edad (años) & & $60.4 \pm 10.5$ & $54.6 \pm 10.1$ \\
Estado civil & & Casado $82.9 \%$ & Casado $80.5 \%$ \\
Escolaridad & $5.9 \pm 2.8$ & $4.9 \pm 3.5$ \\
Ocupación & & \\
& & $56 \%$ & $57 \%$ \\
& Empleadas & $31 \%$ & $29 \%$ \\
& Amas de casa & & $7.0 \pm 3.5$ \\
\hline
\end{tabular}


El grupo control se constituyó por (51.2\%) femenino con edad promedio $54.6 \pm 10.1$ años, el estado civil: (80.5\%) casadas, con una escolaridad promedio de $4.9 \pm 3.5$ años, la ocupación se distribuyó entre empleadas (57\%) y amas de casa (29\%). Con promedio de duración de la enfermedad de $7.1 \pm 3.5$ años (Tabla 1).

Tabla 2. Comparación de las variables de control metabólico entre antes y después, según grupos intervención y control. Pachuca Hidalgo México, 2007- 2008.

\begin{tabular}{lcccccccc}
\hline & \multicolumn{3}{c}{ Grupo intervención } & \multicolumn{5}{c}{ Grupo control } \\
\cline { 2 - 9 } Variables & $\overline{\mathbf{X}}$ & $\mathbf{D E}$ & $\mathbf{t}$ & $\mathbf{P}$ & $\overline{\mathbf{X}}$ & $\mathbf{D E}$ & $\mathbf{t}$ & $\mathbf{P}$ \\
\hline IMC $^{*}$ & 29.67 & 4.13 & 4.56 & 0.001 & 29.16 & 4.94 & -.893 & 0.377 \\
IMC $^{* *}$ & 28.77 & 3.75 & & & 29.50 & 4.92 & & \\
Glucemia plasmática $^{*}$ & 205.10 & 236.71 & 2.89 & 0.006 & 174.24 & 87.16 & 2.35 & 0.023 \\
Glucemia plasmática $^{* *}$ & 96.95 & 24.94 & & & 144.59 & 63.68 & & \\
HbA1C $^{*}$ & 7.06 & 1.41 & 9.53 & 0.001 & 7.70 & 1.95 & -.837 & 0.388 \\
HbA1C $^{* *}$ & 5.66 & .997 & & & 7.88 & 1.78 & & \\
\hline
\end{tabular}

* Antes de la intervención ${ }^{* \star}$ Después de la intervención

El promedio de Indice de Masa Corporal (IMC) del grupo intervención, antes de la intervención educativa fue de $29.67 \pm 4.13$ $\mathrm{kg} / \mathrm{m}^{2}$, y después se observó una reducción significativa en relación a la preintervención; $28.77 \pm 3.75 \mathrm{~kg} / \mathrm{m}^{2},(\mathrm{t}=4.56, \mathrm{p}=0.001)$.

En el grupo control no se observó una reducción significativa del IMC antes de la intervención educativa tradicional; $29.16 \pm$ $4.94 \mathrm{~kg} / \mathrm{m}^{2}$, y después fue $29.50 \pm 4.92 \mathrm{~kg} / \mathrm{m}^{2}$ $(\mathrm{t}=-.893, \mathrm{p}=0.377)$.

Considerando la importancia de las variables metabólicas, los resultados revelaron el promedio de glicemia plasmática elevada en el grupo intervención, antes de la intervención educativa fue de $205.10 \pm 236.71 \mathrm{mg} /$ dl y después expresó una variación hasta los parámetros normales y fue estadísticamente significativa de $96.95 \pm 24.94 \mathrm{mg} / \mathrm{dl}(\mathrm{t}=2.89$, $\mathrm{p}=0.006$ ).

La glicemia plasmática en el grupo control antes de la intervención tradicional;
$174.24 \pm 87.16 \mathrm{mg} / \mathrm{dl}$; después tambien mostró reducción significativa de $144.59 \pm 63.68$ $\mathrm{mg} / \mathrm{dl}(\mathrm{t}=2.35, \mathrm{p}=0.023)$.

$\mathrm{La} \mathrm{HbA1c}$, antes de la intervención educativa, del grupo intervención reveló 7.06\% $(\mathrm{DE}=1.41 \%)$ y después el grupo intervención reveló disminución estadísticamente significativa de 5.66\% (DE=0.997\%), $(\mathrm{t}=9.53 \mathrm{p}<0$. 001) (Tabla 2).

La HbA1c en el grupo control antes de la intervención tradicional 7.70\% (DE=1.95\%); después sin mostrar disminución significativa $7.88 \%(\mathrm{DE}=1.78 \%)(\mathrm{t}=-.837 \mathrm{p}<0.388)$.

Como se observa en la Tabla 3 , en cuanto al perfil de lípidos, se apreció promedio de colesterol total antes de la intervención educativa, el grupo intervención mostró 225.49 $\pm 41.83 \mathrm{mg} / \mathrm{dl}$, después, se distinguió por una disminución significativa de $189.37 \pm$ $39.02 \mathrm{mg} / \mathrm{dl}(\mathrm{t}=6.18 \mathrm{p}<.001)$.

En el colesterol total antes de la intervención tradicional el grupo control mostró 
Tabla 3. Comparación de las variables de control metabólico entre antes y después, según grupos intervención y control. Pachuca Hidalgo México, 2007-2008.

\begin{tabular}{lcccccccc}
\hline & \multicolumn{3}{c}{ Grupo intervención } & \multicolumn{5}{c}{ Grupo control } \\
\cline { 2 - 9 } Variables & $\overline{\mathbf{X}}$ & $\mathbf{D E}$ & $\mathbf{t}$ & $\mathbf{p}$ & $\mathbf{\mathbf { X }}$ & $\mathbf{D E}$ & $\mathbf{t}$ & $\mathbf{P}$ \\
\hline Colesterol $^{*}$ & 225.49 & 41.83 & 6.18 & 0.001 & 212.32 & 38.20 & -0.107 & 0.290 \\
Colesterol $^{*}$ & 189.37 & 39.02 & & & 221.04 & 49.99 & & \\
HDL $^{*}$ & 39.51 & 13.92 & 0.254 & 0.80 & 38.83 & 12.22 & -0.206 & 0.045 \\
HDL $^{* *}$ & 38.93 & 8.40 & & & 43.29 & 15.21 & & \\
LDL $^{*}$ & 163.55 & 217.86 & 0.408 & 0.68 & 154.45 & 171.34 & 1.79 & 0.080 \\
LDL $^{* *}$ & 150.28 & 80.23 & & & 108.25 & 41.34 & & \\
Triglicéridos $^{*}$ & 246.44 & 111.67 & 5.52 & 0.001 & 257.37 & 221.25 & 1.39 & 0.170 \\
Triglicérides $^{* *}$ & 170.14 & 62.46 & & & 233.22 & 151.28 & & \\
\hline
\end{tabular}

* Antes de la intervención ${ }^{* *}$ Después de la intervención

$212.32 \pm 38.20 \mathrm{mg} / \mathrm{dl}$ y después no expresó una variación significativa $221.04 \pm 49.99$ $\mathrm{mg} / \mathrm{dl},(\mathrm{t}=-0.107 \mathrm{p}<.0 .290)$.

El promedio de colesterol-HDL, antes de la intervención educativa, en el grupo intervención fué $39.51 \pm 13.92 \mathrm{mg} / \mathrm{dl}$, y después, con una disminución leve pero, no significativa $38.93 \pm 8.40 \mathrm{mg} / \mathrm{dl}(\mathrm{t}=0.254 \mathrm{p}<0.80)$.

El colesterol-HDL, antes de la intervención tradicional, en el grupo control de 38.83 $\pm 12.22 \mathrm{mg} / \mathrm{dl}$, y después evidenció una elevación favorable con diferencia significativa $43.29 \pm 15.21 \mathrm{mg} / \mathrm{dl}(\mathrm{t}=-0.206 \mathrm{p}<0.045)$.

En relación con el LDL-colesterol, el grupo intervención antes de la intervención educativa $163.55 \pm 217.86 \mathrm{mg} / \mathrm{dl}$, después presentó una disminución sin significancia estadística de $150.28 \pm 80.23 \mathrm{mg} / \mathrm{dl}(\mathrm{t}=0.408$, $\mathrm{p}<0.68)$.

El LDL del grupo control antes de la intervención tradicional fue de $154.45 \pm 171.34$ y después bajaron a una media normal con diferencia significativa; $108.25 \pm 41.34(t=1.79$, $\mathrm{p}<0.08)$.

El promedio de triglicéridos del grupo intervención antes de la intervención educa- tiva tendió a subir $246.44 \pm 111.67 \mathrm{mg} / \mathrm{dl}$ y después disminuyeron al final con una diferencia estadísticamente significativa; 170.14 $\pm 62.46 \mathrm{mg} / \mathrm{dl}(\mathrm{t}=5.52, \mathrm{p}<0.001)$.

Los triglicéridos del grupo control antes de la intervención tradicional fue $257.37 \pm$ $221.25 \mathrm{mg} / \mathrm{dl}$; y después el grupo no reveló una disminución significativa; $233.22 \pm$ $151.28 \mathrm{mg} / \mathrm{dl}(\mathrm{t}=1.39, \mathrm{p}<0.17)$ ( Tabla 3$)$.

Con la finalidad de analizar los efectos antes de la intervención educativa entre el grupo de intervención y el grupo control, despues de la intervencion educativa se observó una diferencia significativa $(\mathrm{p}<0.05)$. El grupo intervención alcanzó un $27 \%$, una reducción de al menos $5 \%$ de su peso inicial, el 55\% regularizó la glicemia plasmática en ayuno, HbA1c, colesterol total, LDL y triglicéridos, la diferencia estadística significativa es a favor del grupo de intervención educativa por enfermería. En relación con el comportamiento del grupo control, sólo el $26 \%$ logró normalizar estas variables, sin mostrar diferencias en forma significativa al final de la intervención. 


\section{DISCUSIÓN Y COMENTARIO}

De las variables sociodemográficas, destaca una población constituida de manera predominante por mujeres dedicadas al hogar o jubiladas. Ellas asisten más a las instituciones de salud, probablemente por la flexibilidad de horarios de trabajo; este hecho se asocia al mayor porcentaje de éstas dentro de los programas de prevención (11). La edad, nivel de escolaridad y ocupación son datos que pueden influir en el control metabólico debido al poco o nulo acceso a la información, así como una menor habilidad de lectura, escritura e incluso de lenguaje, condiciones limitantes para el acceso a oportunidades de aprendizaje o conocimiento de la información proporcionada en este tipo de programa $(8,12)$.

En relación con la variable alterada para el IMC, tanto el Grupo Intervención como el Grupo Control, antes y después de la intervención; presentaban sobrepeso y obesidad desde la preintervención; datos epidemiológicos a nivel mundial señalan que el $80 \%$ de los individuos con DT2 muestran obesidad. Tres estudios actuales señalan la relación entre obesidad y DT2 $(8,13)$; la enfermedad es más común en las personas con IMC mayor a $30 \mathrm{~kg} / \mathrm{m}^{2}(18.5 \%)$, está directamente relacionada con IMC (4) y glucemia, hecho que aumenta la resistencia a la insulina. Similares estudios, de dos recientes publicaciones describen datos similares al de nuestro grupo: sobrepeso $36.3 \%, 24.5 \%$ (13) obesos y $40.7 \%$ sobrepeso, $22.2 \%$ obesos (14).

En relación con los promedios de glicemia plasmática en ayuno, el grupo de intervención educativa en pacientes con DT2, al aplicar la intervención educativa, mostró beneficios en la reducción significativa de la glicemia plasmática controlada menor a 100 $\mathrm{mg} / \mathrm{dl}$, dicha intervención en un 55\% contribuye al ajuste terapéutico, y muestra ser una herramienta importante de enfermería para que el paciente con diabetes tome una deci- sión frente a los episodios de hiper e hipoglucemia (9); estos datos han sido referidos con una frecuencia que oscila entre un $3 \mathrm{y}$ $6 \%$, diferente a la encontrada en este estudio (4\%) (15).

Por otra parte, la HbAlc evidenció una reducción significativa en el grupo de intervención; este estudio tiene implicaciones clínicas y epidemiológicas importantes para la práctica clínica y el desarrollo de políticas de salud pública en México. Por cada $1 \%$ de reducción en $\mathrm{HbA} 1 \mathrm{c}$, por un periodo de 10 años, se asocia con una disminución de $21 \%$ en muertes vinculadas con la diabetes, $14 \%$ en infarto de miocardio y $37 \%$ en complicaciones microvasculares $(9,14)$. Está demostrado que mediante la $\mathrm{HbA} 1 \mathrm{c}$ es posible pronosticar el grado de control de la glucemia $(1,2)$. El promedio de las glucemias de las últimas 8-12 semanas, con una HbAlc menor a $7 \%$, es un parámetro para definir a la diabetes controlada. Valores iguales o mayores fueron catalogados como descontrol glucémico (16). Otros estudios $(1,12,14)$ han demostrado que la reducción de la HbA1c a cifras $\leq 7 \%$ disminuye las complicaciones microvasculares de la diabetes, y si se aplica poco después del diagnóstico de diabetes, ese beneficio es a largo plazo (1). Por lo tanto, una meta razonable para muchos adultos es la necesidad de llevar a cabo intervenciones educativas más efectivas en forma sostenida durante un periodo permanente.

Los resultados del perfil lipídico (colesterol total y triglicéridos), antes de la intervención aparecían alterados en ambos grupos, varios estudios han demostrado una fuerte asociación entre la DT2 y dislipidemia (2, $14,17)$, aspecto relevante para la intervención que incluye: cambios en el estilo de vida, actividad física, dieta baja en colesterol y grasas saturadas; actitudes favorables en la reducción de LDL-colesterol y aumento de HDL-colesterol; parámetros importantes para la evaluación del control metabólico, pero aún poco enfatizados en el control del paciente con diabetes $(18,19)$. Por otra par- 
te, cualquier persona en alto riesgo o riesgo moderado alto que tiene el estilo de vida relacionado con factores de riesgo (la obesidad, inactividad física, niveles elevados de triglicéridos y bajos niveles de HDL) es un candidato para modificar estos factores de riesgo $(3,19)$.

Para mejorar el control glucémico, el manejo farmacológico con antidiabéticos orales o insulinas es parte indispensable del tratamiento; según las recomendaciones del grupo de expertos de la Federación Internacional de Diabetes (2), para diagnóstico y tratamiento de la diabetes con concentraciones lípídicas y lipoproteínas plasmáticas elevadas, presentan factores de riesgo macro y microvascular (3). Por lo tanto, la diabetes ya establecida tiene una fuerte asociación a la dislipemia que influye en la elevación de moléculas LDL, la elevación de los triglicéridos y el descenso del HDL, eleva el riesgo de desarrollar enfermedad cardiovascular por su elevada aterogenicidad (19).

El análisis intragrupos de este estudio reveló alteraciones estadísticas relevantes en las variables de IMC, glucemia en ayuno, HbA1C, colesterol total, triglicéridos, antes y después de la intervención. Destacan diferencias significativas en la disminución de las mismas (8). El cuidado de enfermería del paciente con diabetes debe ser integral, organizado e individualizado de acuerdo con sus necesidades, ofreciendo una intervención con un paradigma educativo para obtener habilidades y destrezas a través de un equipo multidisciplinario $(18,20)$. El éxito en el control de la diabetes no sólo depende de los tratamientos médicos, también se basa en una combinación de intervenciones: monitorización de glucosa, plan de alimentación con los componentes adecuados, actividad física y educación para el autocontrol sobre la naturaleza de la diabetes y cómo controlarla. $(1,4)$.

Los resultados del programa de intervención atendido por enfermería con los algoritmos del protocolo de MDE, mostraron mayores posibilidades de un mejor control metabólico; esto dejar ver la importancia de mantener en forma permanente el programa de intervención con la finalidad de prevenir complicaciones crónicas y propiciar estilos de vida saludables que incidan en una buena calidad de vida para estas personas que viven con diabetes.

Por la eficacia demostrada en México y otros países, se recomienda la implementación del protocolo de MDE, a través de un programa de educación permanente dirigido en la consulta de enfermería que incluya evaluación de resultados de forma periódica, capacitación y actualización de los profesionales para una intervención sistematizada y apoyada en evidencia científica.

Sin embargo, en necesario reconocer las limitaciones del estudio: en primer lugar, los resultados provienen de un centro de salud, eso indica que no pueden ser extendidos a otras instituciones de salud públicas; en segundo lugar, el tamaño de la muestra, con los criterios de inclusión únicamente se aceptaron pacientes constantes a dicho centro.

Finalmente, la intervención educativa dirigida por enfermería fue efectiva al disminuir en forma significativa las concentraciones de glicemia en ayunas, especialmente hemoglobina glicosilada, contribuyendo a mejorar el control metabólico, aceptando que el estudio se deriva de un modelo educativo perfectible con perspectivas favorables para convertirse en la base inicial para facilitar el desarrollo de futuros programas permanentes educativos para personas con diabetes mellitus.

\section{REFERENCIAS}

1. American Association of Diabetes Educators. Definitions: Diabetes Education, Diabetes Educator [Internet]. Chicago, IL: AADE; 2010 [citado 15 mayo 2009]. Disponible en: http://www.diabetese- 
ducator.org/DiabetesEducation/Definitions.html

2. Federación Internacional de Diabetes. Plan mundial contra la diabetes 20112012 [Internet]. Bruselas: Federación Internacional de Diabetes; 2011 [citado 07 nov 2011]. 28p. Disponible: http://www. idf.org/sites/default/files/attachments/ GDP-Spanish.pdf

3. Federación Mexicana de Diabetes, A.C. Diabetes en números [Internet]. México, DF: Federación Mexicana de Diabetes; 2009 [citado 07 nov 2009]. Disponible en: http://www.fmdiabetes.org/fmd/pag/ diabetes_numeros.php

4. Aguilar SCA, Gómez DR, Gómez PFJ. La diabetes tipo2 en México: Principales retos y posibles soluciones. Revista de la ALAD [Internet]. 2011 [citado 1 mayo 2012]; 1(4): 148-61. Disponible en: http://www.revistaalad.com.ar/pdfs/ Revista+ALAD_Vol1_No4_2011-4.pdf

5. Olaiz G, Rivera J, Shamah T, Rojas R, Villalpando $\mathrm{S}$ et al. Encuesta Nacional de Salud y Nutrición: Ensanut [Internet]. México: Instituto Nacional de Salud Pública; 2006 [citado 18 enero 2010]. 132p. Disponible en: http://www.insp.mx/ensanut/ensanut2006.pdf

6. Secretaría de Salud. Programa de Acción Específico 2007-2012 Diabetes Mellitus. México, D.F: Secretaría de Salud; 2008. $82 \mathrm{p}$.

7. Mazze RS, Etzwiler DD, Strock E, Peterson K, McClave CR et al. Staged diabetes management. Toward an integrated model of diabetes care. Diabetes Care. 1994; Suppl 1: 56-66.

8. Otero LM, Zanetti ML, Ogrizio MD. El conocimiento del paciente diabético sobre su enfermedad, antes y después de la implementación de un programa de educación en diabetes. Rev Lat Am Enfermagem. 2008; 16(2): 231-37.

9. Barceló A, Peláez M, Rodríguez WL, Pastor VM. La prevalencia de diabetes diagnosticada entre las personas mayo- res de siete ciudades de América Latina y el Caribe: La Salud, Bienestar y Envejecimiento (SABE) del Proyecto. J El envejecimiento de la Salud. 2006; 18(2): 224-39 [citado 18 enero 2010]. Disponible: http://www.paho.org/spanish/gov/ ce/spp/spp40-11-s.pdf.

10. Leyes y códigos de México. Reglamento de la Ley General de Salud en Materia de Investigación para la Salud. Tomo I. México: Porrúa, 2004. 129-139.

11. Andrade F. Estimating diabetes and diabetes-free life expectancy in México and seven major cities in Latin America and the Caribbean. Rev Panam Salud Pública. 2009; 26(1): 9-16.

12. Zanetti ML, Otero LM, Biaggi MV, Santos MA, Peres DS, Guimarães FP. Satisfaction of Diabetes Patients Under Follow-Up in a Diabetes Education Program. Rev Lat Am Enfermagem. 2007; 15(4): 583-89.

13. Díaz-Perera G, Concepción F, Quintana C, Alemany E. Factores de riesgo y enfermedades consecuentes de la aterosclerosis en pacientes diabéticos. Rev. habanera cienc. méd. 2010; 9(3): 313-20.

14. Otero LM, Zanetti ML and Teixeira CR de S. Sociodemographic and clinical characteristics of a diabetic population at a primary level health care center. Rev Lat Am Enfermagem. 2007; 15(Número especial): 768-73.

15. Gack L, Araujo EP, Do Nascimento $\mathrm{MH}$, Calcalho WH. Consulta de enfermería a portadores de diabetes mellitus e hipertensión arterial: la integración de la educación y la práctica de cuidados de la salud . Cienc. enferm. 2010; XVI(2): 1724.

16. UK Prospective Diabetes Study (UKPDS) Group. Intensive blood glucose control with sulphonylureas or insulin compared with conventional treatment and risk of complications in patients with type 2 diabetes (UKPDS 33). Lancet. 1998; 12; 352(9131): 837-53.

17. Narayan KM, Benjamin E, Gregg EW, 
Norris SL, Engelgau MM. Diabetes Translation Research: Where Are We and Where Do We Want To Be? Ann Intern Med. 2004; 140(11): 958-63.

18. Grundy SM, Cleeman JI, Merz CN, Brewer HB Jr, Clark LT, et al. Implications of recent clinical trials for the $\mathrm{Na}$ tional Cholesterol Education Program Adult Treatment Panel III Guidelines. J Am Coll Cardiol. 2004; 44(3): 720-32.
19. Bertomeu-González V, Núñez D, Bertomeu V. Hipertensión arterial y dislipemia asociadas a diabetes mellitus. Papel de los PPAR. Enfoque terapéutico. Rev Esp Cardiol. 2007; 7(Supl.H): 20-8.

20. Rickheim PL, Weaver TW, Flader JL, Kendall DM. Assessment of Group Versus Individual Diabetes Education: a randomized study. Diabetes Care. 2002; 25(2): 269-74. 